\title{
Diversité végétale et valeur de conservation pour la Biodiversité du Parc National du Mont Péko, une aire protégée, menacée de disparition en Côte d'Ivoire.
}

\author{
Zoro Bertin GONE BI1,2, ", Djaha KOUAME4, Inza KONE2,3, Constant Yves ADOU YAO1,2 \\ 1. Laboratoire de Botanique, UFR Biosciences, Université Félix Houphouët-Boigny, 22 BP 582 Abidjan 22, Côte \\ d'Ivoire \\ 2. Centre Suisse de Recherches Scientifiques en Côte d'Ivoire ; 01 BP 1303 Abidjan 01 \\ ${ }^{3}$ Laboratoire de Zoologie, UFR Biosciences, Université Félix Houphouët-Boigny, Côte d'Ivoire \\ 4. UFR Environnement, Université Jean Lorougnon Guédé, BP 150 Daloa, Côte d'Ivoire \\ *Auteur correspondant : zorobertin@gmail.com
}

Original submitted in on $22^{\text {nd }}$ October 2013 Published online at www.m.elewa.org on 30 th November 2013. https://dx.doi.org/10.4314/jab.v71i1.98820

\section{RÉSUMÉ}

Objectif : Cette étude vise à caractériser la végétation du Parc National du Mont Péko en termes de composition floristique et de présence d'espèces à statut particulier ainsi que la valeur du parc pour la conservation.

Méthodologie et résultats : Un échantillonnage de la végétation a été conduit dans trois zones du Parc National du Mont Péko (PNMP), en Côte d'lvoire. Quinze placettes de $400 \mathrm{~m}^{2}$ ont été mises en place. La flore est riche de 384 espèces appartenant à 88 familles. Les familles les plus riches en espèces sont celles des Fabaceae, des Rubiaceae, des Malvaceae et des Moraceae. Parmi les espèces recensées, 74 sont endémiques, rares et menacées d'extinction au plan national, régional ou international. L'étude a montré que quatre des principales valeurs pour la conservation de la biodiversité sont atteintes par la forêt du parc. Conclusion et application : Cette étude met en évidence l'importance de la sauvegarde du Parc National du Mont Péko, une aire protégée montagneuse, fragile et menacée de disparition. Aussi, la présence de nombreuses espèces à statut particulier justifie l'inclusion du parc dans le «Guinean Forests of West Africa Hotspot» d'importance mondiale pour leur biodiversité. Les différentes caractéristiques du parc révélées par cette étude sont des connaissances nécessaires aux plans de gestion.

Mots clés : Biodiversité, valeur de conservation, Mont Péko, Côte d'Ivoire

\begin{abstract}
Objective: This study aims to assess the plant species diversity and the conservation value for biodiversity of the Mont Péko National Park.

Methodology and results: A botanical sampling was conducted in three areas of the Mont Péko National Park (PNMP), Côte d'Ivoire. Fifteen plots of $400 \mathrm{~m}^{2}$ were established. Three hundred eighty-four (384) plant species belonging to 88 families have been recorded. The richest botanical families are the Fabaceae, Rubiaceae, Malvaceae, and Moraceae. Among the recorded species, 74 are endemics, rare and threatened
\end{abstract}


at national, regional or international level. The study shows that four of the main conservation values for biodiversity are realized.

Conclusion and application: The study highlights the importance of safeguarding the Mont Péko National Park, a mountainous protected area, fragile, and threatened. The presence of many species with particular status justifies the inclusion of the park in the "Guinean Forests of West Africa Hotspot" important for their biodiversity. The characteristics revealed by the study are necessary knowledge for the management plans.

Key words: Biodiversity, conservation value, Mont Péko, Côte d'Ivoire

\section{INTRODUCTION}

Depuis plusieurs décennies, les forêts ivoiriennes sont sujettes à diverses pressions anthropiques telles que l'agriculture extensive sur brûlis, les plantations agro-industrielles, les projets de construction d'ouvrages hydroélectriques, l'exploitation incontrôlée des produits forestiers (non ligneux et bois d'œuvre), l'exploitation minière, la chasse et autres activités de développement telles que les implantations de villages dans les aires protégées. L'accroissement rapide de la population a joué un très grand rôle dans la diminution des surfaces forestières du pays et particulièrement l'Ouest ivoirien. Pour sauvegarder la biodiversité des milieux sensibles, la Côte d'Ivoire a adopté, entre 1968 et 1974, une série de lois visant à instaurer des Parcs Nationaux, des Réserves Naturelles et des Forêts Classées (Monza, 1996). Malheureusement, la demande de plus en plus croissante de produits forestiers et de terres cultivables menace toujours l'intégrité de ces aires et leurs ressources. Le Parc National du Mont Péko (PNMP), à l'instar de la Réserve Naturelle

\section{MÉTHODES D'ÉTUDE}

Milieu d'étude. Le Parc National du Mont Péko, situé dans la région Ouest de la Côte d'Ivoire (Figure 1), entre $6^{\circ} 53^{\prime}-7^{\circ} 08^{\prime}$ de latitude Nord et $7^{\circ} 11^{\prime}-7^{\circ} 21^{\prime}$ de longitude Ouest, couvre une superficie de 34000 hectares. Le parc est essentiellement caractérisé par des plateaux à peine vallonnés de 300 à 500 mètres d'altitude et trois sommets bien individualisés, dont le plus élevé est le Mont Péko. La végétation est constituée de forêt humide semi-décidue qui couvre environ $80 \%$ du parc, de forêt dense sempervirente, de savane boisée guinéenne et de végétations
Intégrale des Monts Nimba et du Parc National du Mont Sangbé, fait partie du « Guinean Forests of West Africa Hotspot», l'une des 18 zones tropicales d'importance mondiale pour leur biodiversité (Conservation International, 2001) Le PNMP héberge des espèces à haute valeur de conservation telles Chrysophyllum azaguieanum J.Miège (Sapotaceae), une plante en danger et Picarthates gymnocephalus Lesson 1828 (Picathartidae), une espèce d'oiseau rare. Malheureusement depuis la crise politico-militaire, les populations y ont installé de vastes plantations agricoles, menaçant ainsi l'intégrité du parc tout en réduisant les habitats préférentiels de ces espèces. Le PNMP, depuis sa création en 1968, n'a bénéficié d'aucune étude approfondie de sa diversité biologique. Pour pallier cette insuffisance, une étude botanique pionnière a été conduite en 1999 dans trois zones du Parc. II s'agira pour nous d'évaluer la diversité floristique et la valeur de conservation du Parc National du Mont pour la biodiversité.

secondaires résultant des activités agricoles (Monza, 1996).

Collecte des données. Dans les zones Nord, Sudouest et Sud-est du Parc, des transects de $100 \mathrm{~m}$ de longueur et $20 \mathrm{~m}$ de largeur ont été mis en place. Chacun a été subdivisé en cinq parcelles de $400 \mathrm{~m}^{2}$ (20 x $20 \mathrm{~m}$ ). Dans chacune de ces parcelles, toutes les espèces végétales ont été recensées et seuls les individus d'arbre dont le diamètre (mesuré à hauteur de poitrine, dbh) est supérieur ou égal à $10 \mathrm{~cm}$, ont été dénombrés. 


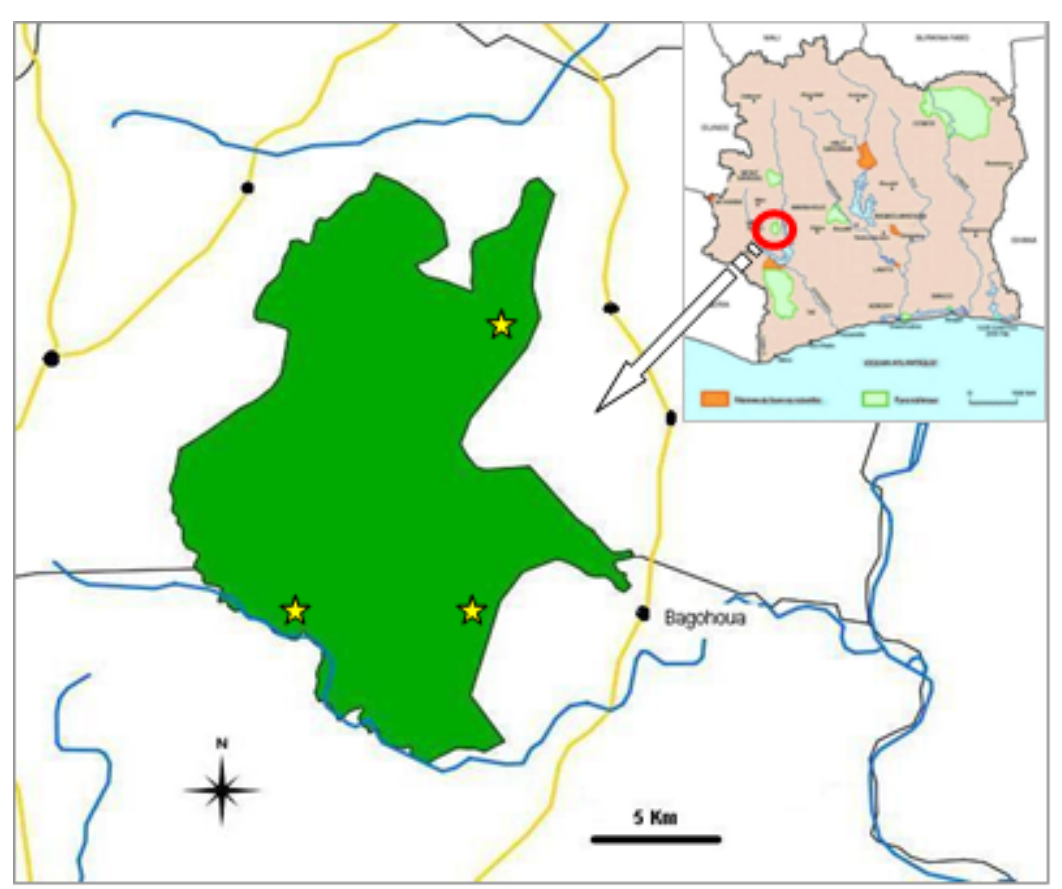

Figure 1. Situation géographique du Parc National du Mont Péko et localisation des zones d'inventaire (

Analyse des Données. L'Herbier national, au Centre National de Floristique de l'Université Félix HouphouëtBoigny et celui du Centre Suisse de Recherches Scientifiques en Côte d'Ivoire (CSRS) ont servi à l'identification des espèces végétales qui n'ont pas pu l'être sur le terrain. Les listes ont été établies selon la classification phylogénétique (APG III, 2009). La richesse spécifique végétale (Aké-Assi, 1984) a été déterminée sans tenir compte de la fréquence, de l'abondance, ni même de la taille et de la productivité des taxons rencontrés (Kouamé, 1998). L'Indice de Shannon (Shannon et Weaver, 1949) a permis d'évaluer la diversité spécifique quantitative selon la formule suivante :

$$
H=-\Sigma(\mathrm{Ni} / \mathrm{N}) \cdot \ln (\mathrm{Ni} / \mathrm{N})
$$

Où Ni est l'effectif de l'espèce i et $\mathrm{N}$ l'effectif total des espèces ;

L'indice permet de tenir compte à la fois du nombre et de l'abondance des espèces. II est utilisé pour apprécier l'hétérogénéité et la diversité d'un biotope (Kouamé et al., 2008 ; Vroh et al., 2010). Les estimateurs Chao 1, Chao 2 (Chao, 1984), Ace (Chazdon et al., 1998) ont permis d'évaluer les richesses spécifiques réelles en tenant compte des espèces rares et moins fréquentes. Les indices et estimateurs ont été calculés à l'aide du logiciel EstimateS (Colwell, 2004). L'équitabilité E de Piélou (Piélou, 1966) a permis d'estimer la régularité de la répartition des individus entre les espèces suivant la formule ci-après : $E=H / l n N$.

La diversité bêta ( $\beta$ ) (qui exprime le taux de remplacement des espèces dans un gradient topographique, climatique, ou d'habitat dans une zone géographique donnée ) estimée à l'aide du coefficient de similitude de Sörensen (Sörensen, 1948) a permis de comparer les richesses spécifiques des trois zones d'inventaire du parc. La formule utilisée est $\mathrm{P}=(2 \mathrm{C} /(\mathrm{A}+$ B)) $x 100$, où $P$ est le coefficient de similitude et exprime le pourcentage d'espèces communes à deux localités comparées, A le nombre total d'espèces de la localité 1 , $B$ le nombre total d'espèces de la localité 2 et $C$ le nombre total d'espèces communes aux deux localités. Valeur de conservation pour la biodiversité. Plusieurs éléments entrent en ligne de compte dans la détermination de la valeur de conservation. Les valeurs attachées à la biodiversité peuvent différer au niveau local, national et international. Dans la présente étude, la valeur pour la conservation de la biodiversité est déterminée à travers la présence des espèces à statut particulier: espèces endémiques, rares et menacées d'extinction de la flore du parc (Aké-Assi, 1988; Jongkind, 2004 ; UICN, 2012). Le concept de Haute valeur pour la conservation (HVC) a été initiée par Forest Stewardship Council (FSC) (Jennings et al., 2003). Six principales valeurs pour la conservation ont été identifiées (Jennings et Jarvie, 2000) avec pour chaque cas, des illustrations qui permettent de conclure 
que ces valeurs sont atteintes pour une forêt donnée. Les critères ont été passés en revue pour rechercher

\section{RÉSULTATS}

Diversité qualitative: Parmi les espèces recensées dans les trois zones du Parc National du Mont Péko, 40 (soit 10,46\%) sont reconnues comme endémiques Ouest Africaines (GCW) dont trois espèces endémiques ivoiriennes (GCi). Trente et une (31) espèces de la liste (soit 8,07 \%) sont reconnues comme endémiques de la Haute Guinée, 30 espèces (soit 7,81\%) sont présentes sur la liste rouge de I'UICN. Parmi elles, une est citée comme rare et menacée d'extinction, 18 sont ceux qui sont réalisés pour le Parc National du Mont Péko.

vulnérables, 3 sont quasi menacées et les 8 autres sont considérées comme étant de préoccupation mineure. Dix (10) espèces de la flore du PNMP (soit 2,60\%) appartiennent à la liste des plantes rares et en voie d'extinction de Côte d'lvoire dont l'une, Maytenus undata (Celastraceae), est une «plante disparue ou ne se rencontrant qu'exceptionnellement » (Tableau 1).

Tableau 1: Liste des espèces à statut particulier rencontrées dans le Parc National du Mont Péko.

\begin{tabular}{|c|c|c|c|c|c|c|}
\hline $\mathrm{N}^{\circ}$ & Espèces & Familles & Chorologie & HG & UICN & $\mathrm{AA}$ \\
\hline 1 & Albizia adianthifolia (Schum.) W.Wight & Fabaceae & & & LC & \\
\hline 2 & Albizia ferruginea (Guill. \& Perr.) Benth. & Fabaceae & & & VU & \\
\hline 3 & Aptandra zenkeri Engl. & Olacaceae & & & & $\mathrm{AA}$ \\
\hline 4 & Baphia nitida Lodd. & Fabaceae & & & LC & \\
\hline 5 & Berlinia occidentalis Keay & Fabaceae & GCW & $H G$ & VU & \\
\hline 6 & Brillantaisia lamium Benth. & Acanthaceae & & & LC & \\
\hline 7 & Bussea occidentalis Hutch. & Fabaceae & & $H G$ & & \\
\hline 8 & Callichilia subsessilis (Benth.) Stapf & Apocynaceae & & $H G$ & & \\
\hline 9 & Calpocalyx brevibracteatus Harms & Fabaceae & & $H G$ & & \\
\hline 10 & Chrysophyllum azaguieanum J.Miège & Sapotaceae & GCW & $H G$ & EN & $\mathrm{AA}$ \\
\hline 11 & Chrysophyllum taiense Aubrév. \& Pellegr. & Sapotaceae & $\mathrm{GCi}$ & $H G$ & & \\
\hline 12 & Cissus doeringii Gilg \& M.Brandt & Vitaceae & & & LC & \\
\hline 13 & Clappertonia minor (Baill.) Bech. & Malvaceae & GCW & & & \\
\hline 14 & Cola caricifolia (G.Don) K.Schum. & Malvaceae & GCW & $H G$ & & \\
\hline 15 & Cordia platythyrsa Baker & Boraginaceae & & & VU & \\
\hline 16 & Costus deistelii K.Schum. & Costaceae & GCW & $H G$ & & \\
\hline 17 & Crossostemma laurifolium Planch. ex Benth. & Passifloraceae & GCW & $H G$ & & \\
\hline 18 & Cryptosepalum tetraphyllum (Hook.f.) Benth. & Fabaceae & GCW & $H G$ & VU & \\
\hline 19 & Cynometra ananta Hutch. \& Dalziel & Fabaceae & GCW & $H G$ & & \\
\hline 20 & Cyperus tenuiculmis Boeckeler & Cyperaceae & & & LC & \\
\hline 21 & $\begin{array}{l}\text { Dactyladenia scabrifolia (Hua) Prance \& } \\
\text { F.White }\end{array}$ & Chrysobalanaceae & & $H G$ & & $\mathrm{AA}$ \\
\hline 22 & Daniellia thurifera Benn. & Fabaceae & GCW & $H G$ & & \\
\hline 23 & Dasylepis brevipedicellata Chipp & Achariaceae & GCW & & & \\
\hline 24 & Dialium aubrevillei Pellegr. & Fabaceae & GCW & $H G$ & & \\
\hline 25 & Dicranolepis persei Cummins & Thymelaeaceae & GCW & $H G$ & & \\
\hline 26 & Dictyophleba leonensis (Stapf) Pichon & Apocynaceae & GCW & & & \\
\hline 27 & Diospyros heudelotii Hiern & Ebenaceae & GCW & & & \\
\hline
\end{tabular}




\begin{tabular}{|c|c|c|c|c|c|}
\hline 28 & Dolichos tonkouiensis Porteres & Fabaceae & GCW & HG & \\
\hline 29 & Dracaena cristula W.Bull & Asparagaceae & GCW & & \\
\hline 30 & Drypetes afzelii (Pax) Hutch. & Putranijvaceae & GCW & & VU \\
\hline 31 & Entandrophragma candollei Harms & Meliaceae & & & VU \\
\hline 32 & $\begin{array}{l}\text { Entandrophragma cylindricum (Sprague) } \\
\text { Spraque }\end{array}$ & Meliaceae & & & VU \\
\hline 33 & $\begin{array}{l}\text { Eribroma oblongum (Mast.) Pierre ex A. } \\
\text { Chev. }\end{array}$ & Malvaceae & & & VU \\
\hline 34 & $\begin{array}{l}\text { Eugenia leonensis Engl. \& Brehmer } \\
\text { Eugenia salacioides G.Lawson ex Hutch. \& }\end{array}$ & Myrtaceae & GCW & & \\
\hline 35 & Dalziel & Myrtaceae & GCW & HG & \\
\hline 36 & Garcinia afzelii Engl. & Clusiaceae & & & VU \\
\hline 37 & Garcinia kola Heckel & Clusiaceae & & & VU \\
\hline 38 & Gomphia schoenleiniana Klotzsch & Ochnaceae & GCW & & \\
\hline 39 & Guibourtia ehie (A.Chev.) J.Leonard & Fabaceae & & & VU \\
\hline 40 & Hibiscus comoensis A.Chev. ex Hutch. \& & Malvaceae & 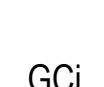 & $H G$ & \\
\hline 41 & $\begin{array}{l}\text { Hymenocardia lyrata Tul. } \\
\text { Irvingia gabonensis (Aubry-Lecomte ex }\end{array}$ & $\begin{array}{l}\text { Phyllanthaceae } \\
\text { Irvingiaceae }\end{array}$ & GCW & $H G$ & \\
\hline 42 & O'Rorke) Baill. & & & & $L R / n t$ \\
\hline 43 & Khaya anthotheca (Welw.) C.DC. & Meliaceae & & & VU \\
\hline 44 & Khaya grandifoliola C.DC. & Meliaceae & & & VU \\
\hline 45 & Lychnodiscus dananensis Aubrév. \& Pellegr. & Sapindaceae & GCW & & \\
\hline 46 & Macaranga beillei Prain & Euphorbiaceae & $\mathrm{GCi}$ & $H G$ & vU \\
\hline 47 & Macaranga heterophylla (Mül.Arg.) Mül.Arg. & Euphorbiaceae & & $H G$ & \\
\hline 48 & Maytenus undata (Thunb.) Blakelock & Celastraceae* & & & \\
\hline 49 & Milicia excelsa (Welw.) C.C.Berg & Moraceae & & & $L R / n t$ \\
\hline 50 & Milicia regia (A.Chev.) C.C.Berg & Moraceae & & $H G$ & VU \\
\hline 51 & Millettia zechiana Harms & Fabaceae & & & LC \\
\hline 52 & Monocymbium deightonii C.E.Hubb. & Poaceae & GCW & & \\
\hline 53 & Myrianthus libericus Rendle & Urticaceae & GCW & $H G$ & \\
\hline 54 & Nauclea diderrichii (De Wild.) Merr. & Rubiaceae & & & Vu \\
\hline 55 & Nemum bulbostyloides (S.S.Hooper) J.Raynal & Cyperaceae & GCW & & \\
\hline 56 & Nesogordonia kabingaensis var. kabingaensis & Malvaceae & & & VU \\
\hline 57 & Pavetta platycalyx Bremek. & Rubiaceae & GCW & HG & \\
\hline 58 & Premna hispida Benth. & Lamiaceae & GCW & & \\
\hline 59 & Psychotria spathacea (Hiern) Verdc. & Rubiaceae & GCW & HG & \\
\hline 60 & Pterygota macrocarpa K.Schum. & Malvaceae & & & VU \\
\hline 61 & Rinorea kibbiensis Chipp & Violaceae & GCW & & \\
\hline 62 & Rinorea longicuspis Engl. & Violaceae & GCW & & \\
\hline 63 & Salacia leonensis Hutch. \& M.B.Moss & Celastraceae & GCW & & \\
\hline 64 & Scadoxus multiflorus (Martyn) Raf. & Amaryllidaceae & & HG & \\
\hline
\end{tabular}




\begin{tabular}{|c|c|c|c|c|c|c|}
\hline 65 & Spermacoce ivorensis Govaerts & Rubiaceae & GCW & & & \\
\hline 66 & Streptocarpus nobilis C.B.Clarke & Gesneriaceae & & & & AA \\
\hline 67 & Strychnos cuminodora Leeuwenb. & Loganiaceae & GCW & & & \\
\hline 68 & Tabernaemontana africana Hook. & Apocynaceae & & $H G$ & & \\
\hline 69 & Trichilia ornithothera J.J.de Wilde & Meliaceae & GCW & $H G$ & VU & \\
\hline 70 & Triplochiton scleroxylon K.Schum. & Malvaceae & & & $\mathrm{LR} / \mathrm{lc}$ & \\
\hline 71 & Urera robusta A.Chev. & Urticaceae & GCW & $H G$ & & \\
\hline 72 & Vepris suaveolens (Engl.) Mziray & Rutaceae & GCW & & $\mathrm{LR} / \mathrm{nt}$ & \\
\hline 73 & Xylia evansii Hutch. & Fabaceae & GCW & $H G$ & & \\
\hline 74 & Xylopia villosa Chipp & Annonaceae & & $H G$ & & \\
\hline & & Total & 40 & 31 & 30 & 10 \\
\hline
\end{tabular}

Diversité quantitative: Au total, 3284 individus de plantes repartis entre 384 espèces ont été dénombrés. Les compositions et proportions par zone sont répertoriées dans le Tableau 2. Parmi les 88 familles botaniques répertoriées dans le parc, les plus riches en espèces sont représentées sur le spectre de la figure 2. Trente-cinq des 88 familles sont monospécifiques et 18 bispécifiques.

Tableau 2 : Nombre d'individus, d'espèces, de familles et les divers indices de diversité dans les trois zones du Parc National du Mont Péko étudiées.

\begin{tabular}{|c|c|c|c|c|}
\hline & Nord & Sud-est & Sud-ouest & Total \\
\hline Nombre d'espèces observées & 203 & 154 & 166 & 384 \\
\hline Nombre de familles & 69 & 55 & 54 & 88 \\
\hline ACE $( \pm S D)$ & $316,9 \pm 30,3$ & $273,8 \pm 23,8$ & $747,1 \pm 311,9$ & $561,3 \pm 42.7$ \\
\hline Chao 1 ( $\pm \mathrm{SD})$ & $420,9 \pm 72,7$ & $280,4 \pm 47,7$ & $677,9 \pm 202,0$ & $691,7 \pm 75.9$ \\
\hline Chao $2( \pm S D)$ & $478,0 \pm 64,6$ & $530,5 \pm 112,8$ & $366,3 \pm 53,6$ & $657,8 \pm 49.2$ \\
\hline Nombre de Singletons & 84 & 60 & 91 & 143 \\
\hline Nombre de Doubletons & 15 & 13 & 7 & 32 \\
\hline Nombre d'Uniques & 160 & 127 & 121 & 229 \\
\hline Nombre de Duplicates & 36 & 16 & 28 & 88 \\
\hline Nombre d'individus & 771 & 1423 & 1090 & 3284 \\
\hline Richesse du peuplement & 3,80 & 9,24 & 6,57 & 8,55 \\
\hline Alpha-Fisher & 89,82 & 43,88 & 54,54 & 112,77 \\
\hline Shannon & 4,99 & 4,39 & 4,47 & 5,24 \\
\hline Equitabilité & 0,94 & 0,87 & 0,87 & 0,88 \\
\hline
\end{tabular}




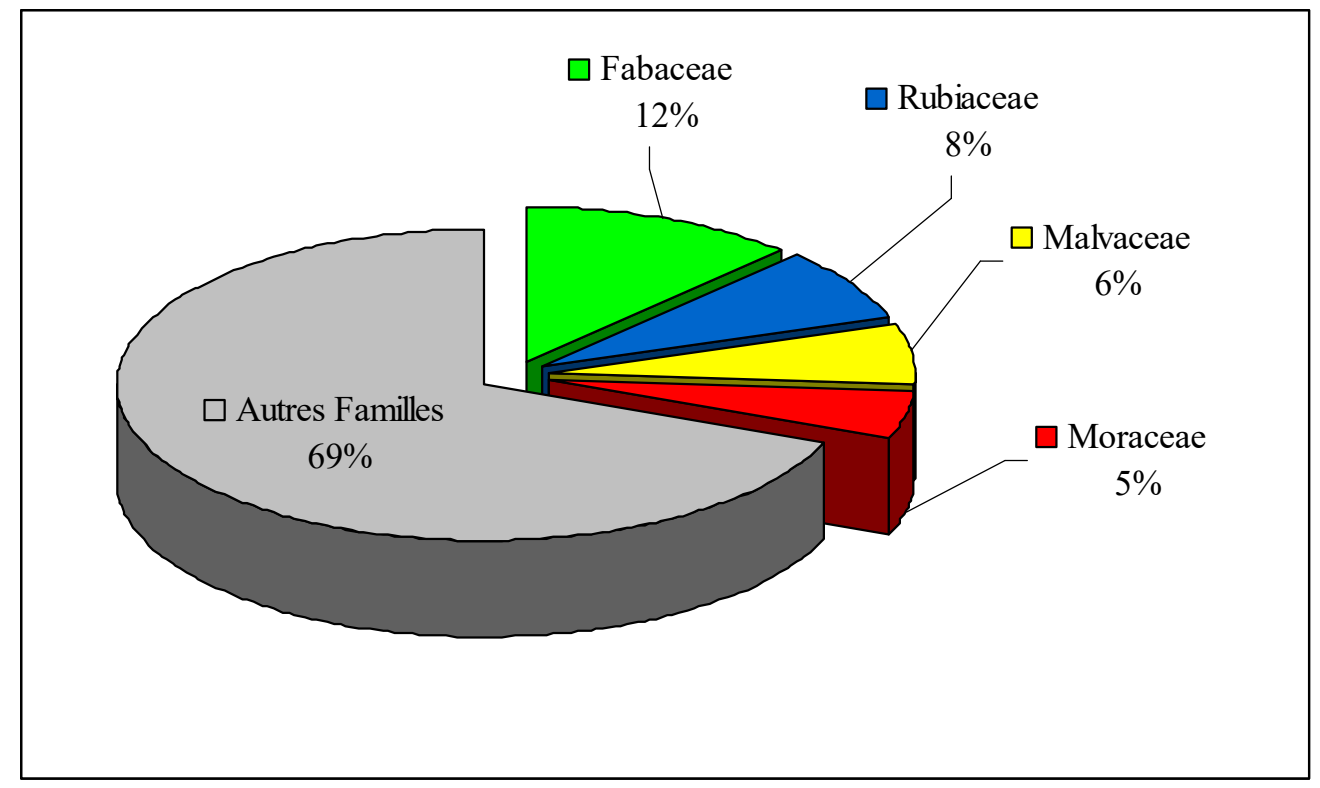

Figure 2: Spectre des familles botaniques inventoriées dans le Parc National du Mont Péko

Les courbes aire-espèces de l'ensemble des placettes réalisées dans le parc (Figure 3 ) reste toujours croissante, montrant que l'aire minimale n'est pas encore atteinte

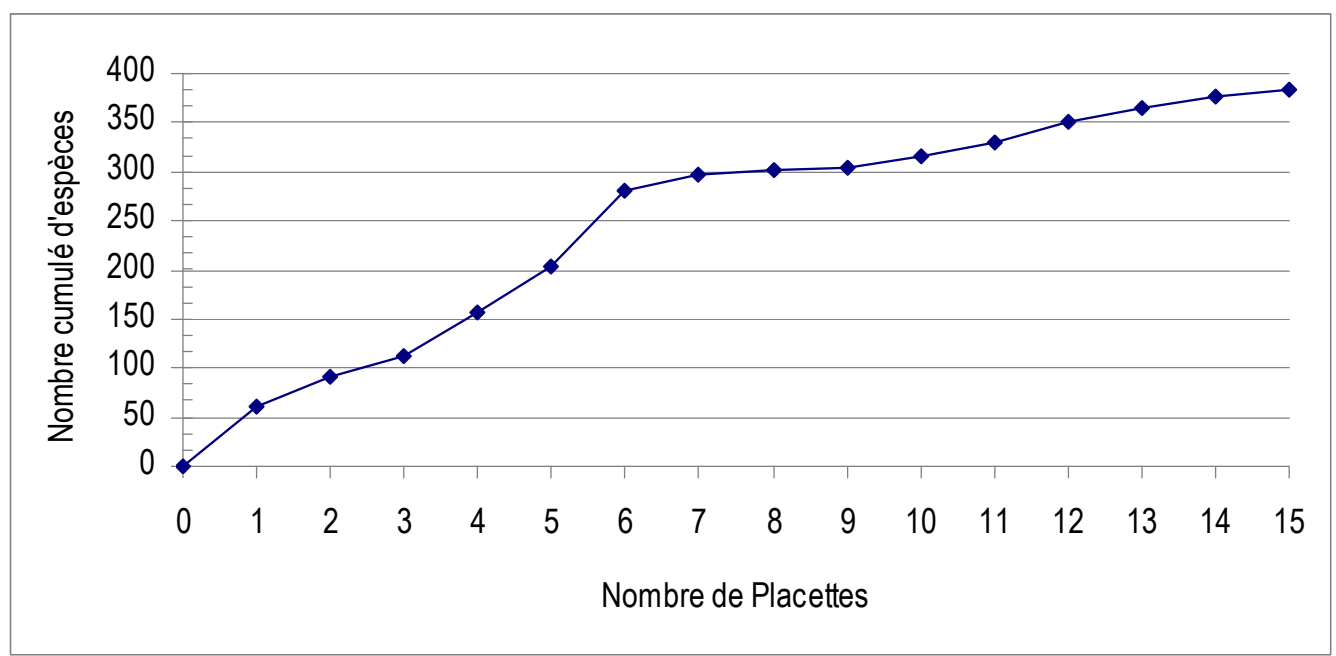

Figure 3 : Courbe aires-espèces de l'inventaire réalisé dans le PNMP.

Les valeurs des coefficients de similarité entre les trois zones d'échantillonnage sont représentées dans le tableau 3. Ces valeurs sont très inférieures à $50 \%$ indiquant ainsi que les trois zones étudiées sont floristiquement différentes les unes des autres.

Tableau 3 : Comparaison des coefficients de similitude de Sorensen des zones d'étude

\begin{tabular}{cccc}
\hline & Nord & Sud-Ouest & Sud-Est \\
\hline Nord & 1 & 0.293 & 0.297 \\
Sud-Ouest & & 1 & 0.369 \\
Sud-Est & & & 1 \\
\hline
\end{tabular}




\section{DISCUSSION}

Diversité botanique de la flore du Parc National du Mont Péko : Les analyses quantitative et qualitative de la flore du Parc National du Mont Péko ont montré que celle-ci est diversifiée à plusieurs niveaux. Les familles botaniques les plus dominantes sont les Fabaceae, les Rubiaceae, les Malvaceae et les Moraceae. Cette situation n'est pas spécifique au Parc National du Mont Péko. En effet, la majorité des forêts ivoiriennes est dominée par le même cortège de familles (Kouamé et al., 1998; Nusbaumer, 2005; N'Da et al., 2008). L'abondance des Rubiaceae dans la flore du PNMP indiquerait, selon le postulat de Guillaumet et Adjanohoun (1971) que celle-ci n'a pas encore atteint le stade climacique. Le nombre élevé d'espèces recensées par cette étude est, cependant, loin de refléter toute la réalité, compte tenu, d'une part, de l'utilisation d'une seule méthode d'inventaire et, d'autre part, de la faible surface échantillonnée. L'association de plusieurs méthodes d'inventaire permet, en effet, d'obtenir des listes floristiques plus grandes même quand les superficies sont moins importantes (Kokou et al., 2005 ; Vroh et al., 2010). En ce qui concerne les espèces à statut particulier, leur présence, leur nombre et leur variété, confirme bien le rôle de conservation de la biodiversité que jouent les aires protégées, en général, et celle du Parc National du Mont Péko, en particulier. La richesse en espèces endémiques, rares et menacées d'extinction justifierait également l'appartenance de la forêt de PNMP au «Guinean Forests of West Africa Hotspot».

Tableau 4 - Richesse floristique, diversité générique et diversité familiale de quelques Forêts Classées (FC) et Parc Nationaux (PN) de Côte d'Ivoire.

\begin{tabular}{llllll}
\hline Région & Auteur & $\begin{array}{l}\text { Surface } \\
\left(\mathbf{K m}^{2}\right)\end{array}$ & $\begin{array}{l}\text { Nombre } \\
\text { d'espèces }\end{array}$ & $\begin{array}{l}\text { Nombre de } \\
\text { genres }\end{array}$ & $\begin{array}{l}\text { Nombre de } \\
\text { familles }\end{array}$ \\
\hline Côte d'Ivoire & Aké-Assi (1998) & 322460 & 3853 & 1270 & 195 \\
PN de la Marahoué & N'da et al. (2008) & 1010 & 607 & 402 & 95 \\
PN du Mont Péko & présente étude & 340 & 384 & $\mathbf{2 8 0}$ & $\mathbf{8 8}$ \\
FC Haut-Sassandra & Kouamé et al. (1998) & 1024 & 1047 & 538 & 114 \\
FC Scio & Nusbaumer et al. (2005) & 18 & 536 & 330 & 91 \\
\hline
\end{tabular}

Le tableau 4 présente les résultats de diversité (espèces, genres et familles) dérivant de la liste floristique, en comparaison avec des études menées dans des forêts denses semi-décidues proches ou situées sur la même latitude que le parc à différentes échelles. Ces données sont uniquement fournies à titre indicatif et aucune conclusion ne saurait être tirée de ces chiffres en raison de la nature différente des études. Valeur et implication pour la conservation de la biodiversité: Le concept de Haute valeur pour la conservation (HVC) vise à s'assurer que les plus importantes valeurs pour la conservation sont gérées correctement dans le contexte de certification forestière prônée par la Forest stewardship council (FSC) (Jennings et al., 2003). Des six principales valeurs pour la conservation, les suivantes peuvent être retenues pour le Parc National du Mont Péko :

- HVC 1 : zones forestières contenant des concentrations de valeurs de biodiversité significatives au plan global, régional ou national. Les résultats présentés ici montrent que cette valeur se retrouve en partie atteinte pour le Parc National du Mont Péko, vu qu'il regorge d'espèces végétales à statut particulier telles que les espèces endémiques (49), menacées (30) et rares (10). Au niveau faunistique, des traces d'activités d'éléphants, de félins, de buffles et de céphalophes ont été observées.

- HVC 3 : zones forestières qui sont comprises ou qui contiennent des écosystèmes rares, menacées ou en danger. Les Parcs des monts Péko et Sangbé ainsi que la réserve des Monts Nimba sont les seules aires protégées occupant des écosystèmes fragiles montagneux (Adou Yao et al., 2011). Ces derniers sont aujourd'hui très menacés par des activités anthropiques croissantes. La présente valeur est de ce fait entièrement atteinte pour le PNMP.

- HVC 4 : zones forestières qui procurent des services de base en matière de protection de la nature (ceci inclut la protection des sources d'eau, protection contre l'érosion et le feu destructeur). Dans le PNMP, de nombreux ruisseaux prennent leur source. De plus les formations sempervirentes de sa végétation ainsi que l'abondante litière humide contribuent 
à freiner l'avancement de feux de forêt et minimisent les phénomènes d'érosion. La valeur HVC 4 se trouve donc atteinte.

- HVC 5 : zones de forêts fondamentales aux besoins des communautés locales (par exemples subsistance, santé). La chasse pratiquée dans ce parc contribue beaucoup dans l'approvisionnement en protéine animale

\section{CONCLUSION}

Cette étude a permis de poser la base des connaissances sur la flore du Parc National du Mont Péko (PNMP). Elle a montré que la forêt du PNMP abrite un important nombre d'espèces végétales appartenant à plusieurs familles botaniques. La grande diversité de la flore du parc est aussi reflétée dans la présence de plusieurs espèces à statut particulier telles que les espèces endémiques (ivoiriennes, ouest africaines et de Haute Guinée) et espèces menacées et rares (surtout celles de la liste rouge de l'UICN). La diversité de la flore du parc révélée par la présente

\section{REFERENCES BIBLIOGRAPHIQUES}

Adou Yao CY, N'Da DH, Bakayoko A, Kouassi KE, N'Guessan KE, Aké-Assi L, 2011. Distribution, Regeneration and Conservation Status of Nuxia congesta $\mathrm{R}$. Br. ex Fresen. (Buddlejaceae) in Côte d'Ivoire. American Journal of Scientific and Industrial Research, 2 (5): 774-785.

Aké Assi, L., 1998. Impact de l'exploitation forestière et du développement agricole sur la conservation de la biodiversité biologique en Côte d'Ivoire. Le flamboyant, 46, pp 20-22.

Aké-Assi L, 1988. Espèces rares et en voie d'extinction de la flore de la Côte d'Ivoire. Monographie Syst. Bot. Missouri Botanical Garden. 25: 461 $-463$.

Aké Assi L, 1984. Flore de la Côte d'Ivoire : Etude descriptive et biogéographique avec quelques notes ethnobotaniques, Thèse de Doctorat d'État, Faculté de Sciences et Techniques, Université Nationale d'Abidjan, Côte d'Ivoire, $1206 \mathrm{p}$.

Angiosperm Phylogeny Group, 2009. An update of the angiosperm phylogeny group classification for the orders and families of flowering plants: APG III Botanical Journal of Linnean Society 141: 399-436.

Chao A, 1984. Nonparametric estimation of the number of classes in a population. Scandinavian des populations riveraines qui y pénètrent régulièrement de façon illégale. Aussi, le prélèvement, dans le PNMP, de plusieurs espèces végétales qui sont utilisées dans l'alimentation, la médecine traditionnelle, la construction d'habitats et même la fabrication d'objets de culte tels les masques, montre que la valeur HVC 5 est atteinte.

étude justifie son inclusion dans le « Guinean Forests of West Africa Hotspot» défini par Conservation International. Au plan des valeurs de conservation, quatre des six catégories définies par la FSC sont atteintes entièrement ou partiellement dans le parc, ce qui lui vaut le statut de parc à haute valeur de conservation. L'étude a montré également que cet écosystème fragile et les ressources qu'il contient sont menacés. II est donc urgent que des études ultérieures à la présente y soient conduites pour évaluer son état actuel de conservation.

\section{Journal of Statistics 11: 265-270.}

Chazdon RL, Colwell RK, Denslow JS, Guariguata MR, 1998. Statistical methods for estimating species richness of woody regeneration in primary and secondary rain forests of $\mathrm{NE}$ Costa Rica. In Forest Biodiversity Research, Monitoring and Modeling: Conceptual Background and Old World Case Studies, F. Dallmeier and J. Comiskey (eds), 285-309. Paris, France: Parthenon Publishing.

Colwell RK; 2004. EstimateS: Statistical estimation of species richness and shared species from samples.téléchargeable sur [http ://viceroy.eeb.uconn.edu/EstimateS]..

Conservation International, 2001. De la Forêt à la Mer : les Liens de Biodiversité de la Guinée au Togo. Conservation International. Washington DC

Guillaumet JL. et Adjanohoun E, 1971. La végétation de la Cote d'Ivoire. In Le milieu naturel de Côte d'Ivoire. Avenard, J.-M., M., Eldin, G., Girard, J., Sircoulon, P., Touchebeuf, J.-L., Guillaumet, E., Adjanohoun et A., Perraud, Mémoires ORSTOM n ${ }^{\circ}$ 50, Paris , France, pp 161-263.

Jennings $S$ et Jarvie J, 2000. A Sourcebook for Landscape Analysis of High Conservation Value Forests Version 1, ProForest, $50 \mathrm{p}$. 
Jennings S, Nussbaum R, Judd J, Evans T, 2003. The High Conservation Value Forest Toolkit Edition 1, ProForest, 27p.

Jongkind $\mathrm{CCH}, 2004$. Checklist of Upper Guinea forest species In Biodiversity of West African Forests : An Ecological Atlas of Woody Plant Species. Poorter, L. ; F., Bongers, F. N., Kouamé, W. D., Hawthorne, Cabi Publishing, London, pp 447- 477.

Kokou K, Adjossou K, Hamberger K, 2005. Les forêts sacrées de l'aire Ouatchi au sud-est du Togo et les contraintes actuelles des modes de gestion locale des ressources forestières, [VertigO] - la revue électronique en sciences de l'environnement 6 (3). [En ligne], URL : http://vertigo.revues.org/2456. Consulté le 10 septembre 2013.

Kouamé D, Adou Yao CY, Kouassi KE, N'Guessan KE, Akoi K, 2008. Preliminary floristic inventory and diversity in Azagny National Park (Côte d'Ivoire). European Journal of Scientific Research, 23 (4): 537-547.

Kouamé NF, Tra Bi FH, Etien D, Traoré D, 1998. Végétation et flore de la forêt classée du HautSassandra en Côte d'Ivoire. Science et Médecine 00 : 28-35.

Kouamé NF, 1998. Influence de l'exploitation forestière sur la végétation et la flore de la forêt classée du Haut Sassandra (Centre ouest de la Côte d'Ivoire). Thèse Doctorat $3^{\text {ème }}$ cycle, UFR Biosciences, Université Cocody, Abidjan Côte d'Ivoire , $203 \mathrm{p}$.

Monza (de) JP, 1996. L'Atlas pour la conservation des forêts tropicales d'Afrique. UICN, $310 p$.

Pielou EC, 1966. The measurement of diversity in different types of biological collections. Journal of Theoretical Biology 13: 131-144.

N'Da DH, Adou Yao CY, N'Guessan KE, Koné M, Sangne YC, 2008. Analyse de la diversité floristique du Parc National de la Marahoué, Centre-ouest de la Côte d'Ivoire. Afrique SCIENCE 4(3) : 552-579.

Nusbaumer L, Gautier L, Chatelain C, Spichiger R, 2005. Structure et composition floristique de la forêt classée de la Scio (Côte d'Ivoire). Etude descriptive et comparative. Candollea 60(2): 393-443.

Shannon CE et Weaver W, 1949. A mathematical theory of communication. University of Illinois Press.

Sörensen T, 1948. A method of establishing groups of equal amplituin plant sociology based on simililarity of species content. Der Kongelige Danske Videnskabernes Selskab. Biologiste Skrifter 5 (4):1-34.

UICN, 2012. IUCN Red List of Threatened Species, Version 2012, 2, [En ligne], URL: http://www.iucnredlist.org, Consulté le 7 janvier 2013.

Vroh BTA, Adou Yao CY, Kouamé D, N'Da DH, N'Guessan KE, 2010. Diversités Floristique et Structurale sur le Site d'une Réserve Naturelle Volontaire à Azaguié, Sud-est de la Côte d'Ivoire. European Journal of Scientific Research 45 (3): 411-421. 